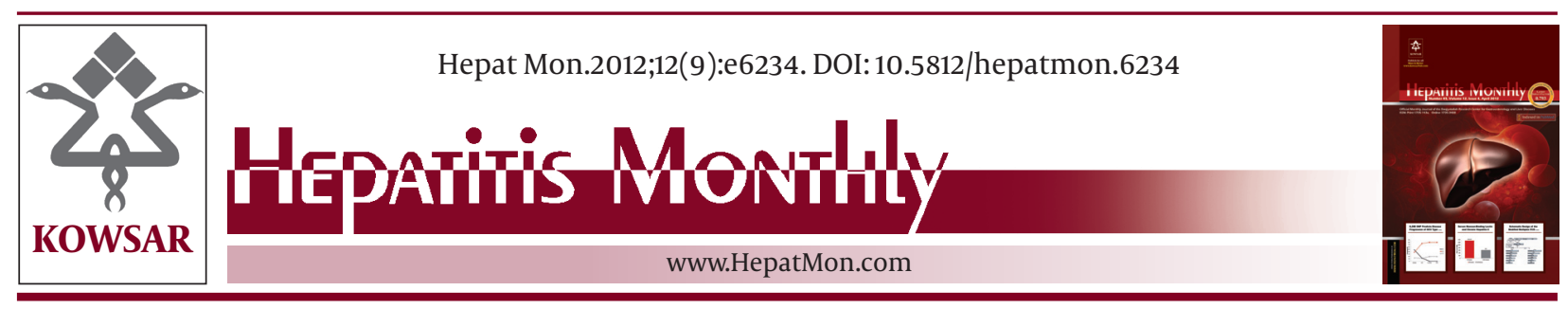

\title{
Effects of Oral Levamisole as an Adjuvant to Hepatitis B Vaccine in HIV/ AIDS Patients: A Randomized Controlled Trial
}

\author{
Babak Sayad ${ }^{1^{*}}$, Seyyed Moayed Alavian ${ }^{2}$, Farid Najafi ${ }^{3}$,Bita Soltani ${ }^{1}$, Maria Shirvani ${ }^{1}$, Alireza \\ Janbakhsh ${ }^{1}$, Feyzollah Mansouri ${ }^{1}$, Mandana Afsharian ${ }^{1}$, Siavash Vaziri ${ }^{1}$, Arash Alikhani ${ }^{1}$, \\ Homayoon Bashiri ${ }^{1}$ \\ ${ }^{1}$ Liver Disease and Hepatitis Research Center, Kermanshah University of Medical Sciences, Kermanshah, IR Iran \\ ${ }^{2}$ Research Center for Gastroenterology and Liver Diseases, Baqiyatallah University of Medical Sciences, Tehran, IR Iran \\ ${ }^{3}$ Health Research Center, Kermanshah University of Medical Sciences, Kermanshah, IR Iran
}

\begin{tabular}{l}
\hline A R T I C L E I N F O \\
\hline Article type: \\
Original Article \\
\hline Article history: \\
Received: 09 May 2012 \\
Revised: 13 Jun 2012 \\
Accepted: 04 Jul 2012 \\
\hline
\end{tabular}

\section{Keywords:}

HIV

Hepatitis B Virus

Vaccination

Levamisole

\begin{abstract}
A B S T R A C T
Background: Human immunodeficiency virus (HIV) infected patients are also frequently exposed to the hepatitis B virus (HBV), due to the common routes of transmission, therefore, prevention of hepatitis B results in decreased complications of the disease. objectives: Since the immune response of HIV patients to hepatitis B vaccination is less robust than that found in healthy individuals, this study aimed to evaluate the effect of a levamisole adjuvant on increasing the immune response.

Patients and Methods: In this study, 89 HIV infected patients, without a history of HBV infection or vaccination, were randomly allocated into experimental (44 patients) and control (45 patients) groups. HBV vaccination was performed using the Hepavax-Gene TF vaccine, $40 \mu \mathrm{g}$ three times at intervals of; zero, one, and three months. Levamisole 50 mg twice a day or a placebo, was administered to the experimental and control groups, respectively, for a period of six days before to six days after the vaccination. Immune response was evaluated by measuring hepatitis B surface antibodies (HBsAb) concurrently with the second and third vaccine administration, and at one and three months at the conclusion of the vaccination program.

Results: The immune response following the threevaccinations was higher in those who were receiving levamisole compared with the controls (90\% vs. 65.38\%) $(P=0.05)$. Furthermore, the immune response and the mean antibody titer following the repeated vaccination in the experimental group showed a higher increase than in the control group. The immune response and the mean titer of antibody were not associated with; age, sex, body mass index, history of smoking and/or intravenous drug use in either of the groups. However, regarding CD4+ cells more than 200 cell $/ \mathrm{mm} 3$, mean antibody production significantly increased in both groups.

Conclusions: Using levamisole with the hepatitis B vaccination can increase the immune response and antibody titer mean in HIV infected patients. Since these patients have a more complete response with CD4+ cells more than $200 \mathrm{cell} / \mathrm{mm} 3$, vaccination and effective adjuvants seem to be most beneficial when CD4+ cells are greater than 200 cell/mm3, in HIV infected patients.

Published by Kowsar Corp, 2012. cc 3.0.
\end{abstract}

Implication for health policy/practice/research/medical education:

The immunization of HIV/AIDS patients against Hepatitis B Virus infection is one of the most important issues confronted by physicians. The results of this study suggest that Levamisole as an adjuvant to hepatitis B vaccine might be considered for increase the immune response to vaccination in these patients.

${ }^{*}$ Corresponding author: Babak Sayad, Liver Disease and Hepatitis Research Center, Kermanshah University of Medical Sciences, Kermanshah, IR Iran. Tel: +988317257708, Fax: +98-8318377734, E-mail: babaksayad@yahoo.com

DOI:10.5812/hepatmon.6234

(C) 2012 Baqiyatallah Research Center for Gastroentrology and liver diseases; Published by Kowsar Corp.

This is an Open Access article distributed under the terms of the Creative Commons Attribution License (http://creativecommons.org/licenses/by/3.0), which permits unrestricted use, distribution, and reproduction in any medium, provided the original work is properly cited. 
- Please cite this paper as:

Sayad B, Alavian SM, Najafi F, Soltani B, Shirvani M, Janbakhsh A, et al. Effects of Oral Levamisole as an Adjuvant to Hepatitis B Vaccine in HIV/AIDS Patients: A Randomized Controlled Trial. Hepat Mon. 2012;12(9):e6234. DOI: 10.5812/hepatmon.6234

\section{Background}

Human immunodeficiency virus (HIV) infected patients are often vulnerable to developing hepatitis B as well, this is due to their common routes of transmission $(1,2)$. In Asia, $34 \%$ to $98 \%$ of HIV infected cases show evidence of a previous hepatitis B infection (3). Moreover, HIV infection has a significant effect on the course of a hepatitis $B$ virus (HBV) infection, causing a three-fold increase in the incidence of permanent antigenemia with hepatitis B surface antigens (HBsAg). Liver-related mortality rates of patients with both HBV and HIV infections show a 10-15 fold increase compared with those with only one of these infections. Also, it has been observed that HIV infected patients who have previously been infected with HBV were more likely to develop chronic disease $(4,5)$ which can result in; cirrhosis, hepatic failure and development of hepatocellular carcinoma $(3,6)$. Therefore, prevention of hepatitis B in HIV infected patients plays an important role in improving the quality and quantity of their lives. It should be noted that more than $95 \%$ of the general population with a normal immune system will have an appropriate response to the standard vaccine dose $(2,7,8)$, however, the immune response in HIV infected patients is reduced, estimated to be about $40 \%$ to $60 \%(2,9)$. This results from the development of the infection and the reduced number and function of CD4+ lymphocytes, as well (10). Regarding the aforementioned issues and the importance of effective vaccination in these patients, many studies have been conducted in order to find more effective methods, for example using intradermal injections instead of intramuscular injections and administering these once a week (11), at double doses (12) or higher doses $(13,14)$ instead of the usual dose and using adjuvants with the vaccination $(1,9)$. Levamisole is one of the adjuvants which have been used widely in the treatment of wormborne diseases. Since 1972 when its stimulatory effect on the immune system was discovered, it has been used for many other diseases as well. This drug can improve the immune system by stimulating macrophage and $\mathrm{T}$ cell functions $(15,16)$. Several studies have used this stimulatory effect, in order to increase immunization following vaccination, thus leading to more effective results (16-18).

\section{Objectives}

The present study aimed to evaluate the effect of levamisole, as an adjuvant for the hepatitis B vaccine, in increasing the immune response in HIV/AIDS patients.

\section{Patients and Methods}

The study population were all HIV/AIDS patients who had therapeutic files in the Behavioral Diseases Consultation Center affiliated to the Health Affairs Deputy of Kermanshah University of Medical Sciences. In coordination with the Health Deputy of the University, the patients who had files in the Behavioral Diseases Consultation Center in whom a HIV infection was confirmed by two positive HIV antibody (HIV Ab) enzyme-linked immunosorbent assay (ELISA) tests and one positive HIV Ab Western blot (WB) test without any history of developing hepatitis B (negative HBsAg, hepatitis B core antibody (HBcAb), and hepatitis B surface antibody (HBsAb)), and immunization against the disease were included in the study. An informed consent form was signed by all participants and the Ethical Committee of Kermanshah University of Medical Sciences approved the study. Using a table of random numbers, the participants were divided into experimental and control groups, each receiving a $40 \mu$ gr Hepavax-Gene TFvaccine(Berna Biotech,India in the deltoid muscle at 0,1 , and 3 months. Six days before each of the vaccinations and continuing until six days afterwards, patients in the experimental group received 50 mgr oral levamisole twice a day, while the controls used placebo tablets instead of levamisole, but in the same order. At the time of the second and third vaccinations and also one and three months after the third vaccination, serum samples were collected to measure the immune response. In order to reduce bias, a systematic random allocation method was used to assign study participants into experimental and control groups, as a result, all of the participants and the interviewers were blind to the allocation method. As we also had access to patients' files, we were able to collect all of the relevant information regarding the patients' baseline characteristics and health status history; age, sex, smoking history, and history of intravenous drug use, history of opium use, antiretroviral therapy and CD4 count. To check the efficacy of the random allocation, we compared baseline characteristics of the patients and their health profiles with a two independent samples t-test (for continuous variables) and a chi-squared test (for qualitative variables). We used a chi-squared test and two independent sample t-tests, in order to compare the level of immune response and $\mathrm{Ab}$ titer, respectively. The level of significance was defined as less than 0.05. Although we excluded patients who were missed during follow-up, we analyzed the data using an intention-to-treat analysis.

\section{Results}

Among the 150 patients referred to the center during a period of 24 months (from April 2008 until April 2010), 89 patients met the inclusion criteria, and these individ- 


\begin{tabular}{llll}
\hline \multicolumn{1}{l}{ Table 1. Demographic Data and Distribution of Study Variables in the Experimental and Control Groups } \\
\hline & Levamisole Group $(\mathbf{n}=\mathbf{4 4})$ & Placebo Group $(\mathbf{n}=\mathbf{4 5})$ & P value \\
\hline Female, $\%$ & 15.9 & 31.11 & 0.09 \\
\hline Age, $\mathbf{y}$, Mean \pm SD & $32.23 \pm 7.52$ & $6.34 \pm 31.71$ & 0.66 \\
\hline BMI, $\mathbf{~ k g} / \mathbf{m}^{2}$, Mean \pm SD & $22.8 \pm 2.63$ & $22.28 \pm 2.62$ & 0.32 \\
\hline Positive history of smoking, \% & 84.09 & 80 & 0.61 \\
\hline Positive history of using opium, \% & 63.64 & 66.67 & 0.76 \\
\hline Positive history of intravenous drug use, \% & 72.73 & 71.11 & 0.86 \\
\hline Positive history of antiretroviral treatment, \% & 15.9 & 33.33 & 0.06 \\
\hline CD4, $\mu$ L, Mean \pm SD & $374.2 \pm 209.6$ & $341.46 \pm 219.9$ & 0.5 \\
\hline
\end{tabular}

Abbreviation: BMI, body mass index.

\begin{tabular}{lllll}
\hline \begin{tabular}{l} 
Table 2. Level of Immune Response During the Three Vaccinations in the Experimental and Control Groups \\
\hline
\end{tabular} & Levamisole Group, \% & Placebo Group, \% & P value & OR(95\%CI) \\
\hline $\begin{array}{l}\text { Level of immune response one month after the first } \\
\text { vaccination }(\mathbf{n}=\mathbf{6 1})\end{array}$ & 20.69 & 34.38 & 0.23 & $0.50(0.16-1.60)$ \\
$\begin{array}{l}\text { Level of immune response two months after the } \\
\text { second vaccination }(\mathbf{n}=\mathbf{4 9})\end{array}$ & 55 & 48.28 & 0.64 & $1.3(0.42-4.1)$ \\
$\begin{array}{l}\text { Level of immune response one month after the } \\
\text { third vaccination }(\mathbf{n}=\mathbf{4 3})\end{array}$ & 84.21 & 58.33 & 0.07 & $3.81(0.87-16.7)$ \\
$\begin{array}{l}\text { Level of immune response three months after the } \\
\text { third vaccination }(\mathbf{n}=\mathbf{4 6})\end{array}$ & 90 & 65.38 & 0.05 & $4.76(0.90-25.3)$ \\
\hline
\end{tabular}

Abbreviation: OR, odd's ratio.

\begin{tabular}{llll}
\hline \multicolumn{4}{|l}{ Table 3. hepatitis B Surface Antibody Titer During the Three Vaccinations in the Experimental and Control Groups } \\
\hline & Levamisole Group, Mean \pm SD & Placebo Group, Mean \pm SD & P value \\
\hline HBs-Abtiter one month after the first vaccination $(\mathbf{n}=\mathbf{6 1})$ & $19.14 \pm 9.07$ & $32 \pm 17.35$ & 0.23 \\
\hline HBs-Ab titer two months after the second vaccination $(\mathbf{n}=\mathbf{4 9 )})$ & $38.85 \pm 42.83$ & $36.01 \pm 39.96$ & 0.81 \\
\hline HBs-Abtiter one month after the third vaccination $(\mathbf{n}=\mathbf{4 3})$ & $56.05 \pm 38.16$ & $40.12 \pm 41$ & 0.19 \\
\hline HBs-Abtiter three months after the third vaccination $(\mathbf{n}=\mathbf{4 6})$ & $53.75 \pm 34.49$ & $45.15 \pm 38.85$ & 0.43 \\
\hline
\end{tabular}

Abbreviation: HBsAb, hepatitis B surface antibody.

uals were randomly entered into the experimental (44 patients) and control (45 patients) groups, respectively. Table 1 shows the demographic data and the distribution of the variables in each of the groups. According to the table, the two groups were comparable regarding; age, sex, body mass index, history of smoking, history of using opium, history of intravenous drug use, mean count of CD4+ cells as well as antiretroviral treatment (Table 1). The second vaccination was administered to a total of 61 patients, this was because 28 individuals did not wish to continue their participation, and they did not have the second vaccination. For the third vaccination, only 49 patients received the vaccine as; four patients had died, two patients had emigrated from Kermanshah Province and six did not desire to continue their participation. Among the 49 individuals receiving all three of the vaccinations, 43 patients were referred for the first measurement of $\mathrm{HB}$ sAb titer, while the final (second) titration of HBsAb was performed on 46 patients. The percentage of immune response (HBsAb $\geq 10 \mathrm{IU} / \mathrm{mL}$ ) and mean titer of $\mathrm{HBsAb}$ during the three vaccination times in the experimental and control groups are summarized in Tables 2 and 3. The level of immune response one month after the third vaccination in the experimental group (who received levamisole adjuvant) and the controls (who received placebo) showed an $84.21 \%$ and $58.33 \%$ increase, respectively. However, three months after the third vaccination, immune response had increased to $90 \%$ in the experimental group and $65.38 \%$ in the controls $(P=0.05)$ (Figure 1$)$. The calculated number-needed-to treat for three months after the third vaccination is equal to 4 .

As shown, the level of immune response in the experimental group was significantly higher than in the controls, and this increased with repeated vaccination. Furthermore, the mean titer of HBsAb one month after the third vaccination was $56.05 \mathrm{IU} / \mathrm{mL}$ in the experimental group and $40.12 \mathrm{IU} / \mathrm{mL}$ in the control group, and three months after the third vaccination it had increased to 


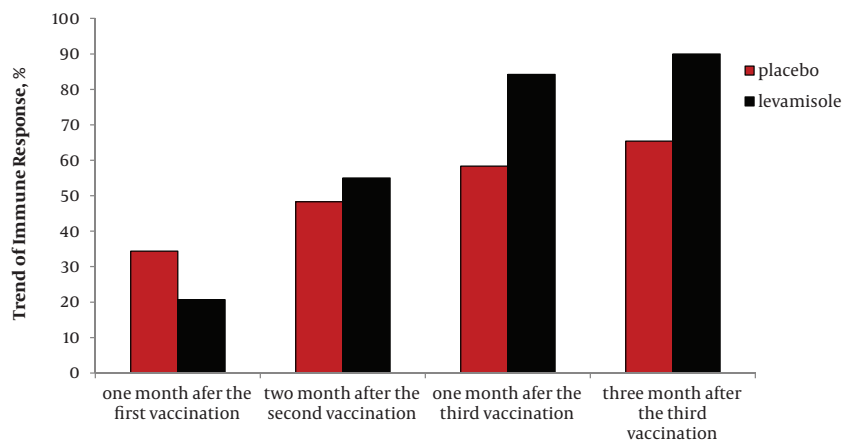

Figure 1. Trend of Immune Response in the Experimental and Control Groups

$53.76 \mathrm{IU} / \mathrm{mL}$ and $45.15 \mathrm{IU} / \mathrm{mL}$ in the experimental and control groups, respectively (Figure 2). According to Figure 2 , the mean antibody production in the experimental group was higher than the control group. The level of immune response one month after the first vaccination was $20.69 \%$ in the experimental group, and $34.38 \%$ in the controls (OR $=0.50,95 \% \mathrm{CI}$ : 0.16-1.6), at the same time, the mean antibody titer was 9.07 and 17.35 in the experimental and the control groups, respectively. The level of immune response two months after the second vaccination was 55\% in the experimental group and $48.28 \%$ in the controls ( $\mathrm{OR}=1.31,95 \% \mathrm{CI}$ : 0.42-4.11) while the antibody titer was reported to be 38.85 and 36.01 in the experimental and control groups, respectively (Figure 1 and 2). None of the variables of age or body mass index had a significant association with antibody production $(P>0.05)$. Moreover, the effect of the study variables including; sex, age, body mass index, history of smoking, history of opium use, history of intravenous drug use, history of receiving antiretroviral drugs, and mean count of CD4+ cells, on the trend of immune response and mean antibody titer were evaluated separately. These variables had no associations with immune response and mean antibody titer $(P>0.05)$, however, the mean count of CD4+ cells higher than $200 \mathrm{cell} / \mathrm{mm} 3$ significantly affected mean antibody production one month and three months after the third vaccination.

\section{Discussion}

As a result of high risk behaviors, the majority of HIV infected patients have an increased chance of developing hepatitis B (3). In addition, HBV infection in this group of patients is also associated with more complications and a higher mortality rate. Through means of inducing immunodeficiency, HIV infections result in the chronicity of HBV infection and this facilitates virus proliferation in the liver, resulting in an acceleration in and exacerbation of liver destruction $(9,10)$. It should be noted that the use of antiretroviral drugs and controlling opportunistic infections in HIV positive patients has resulted in longer

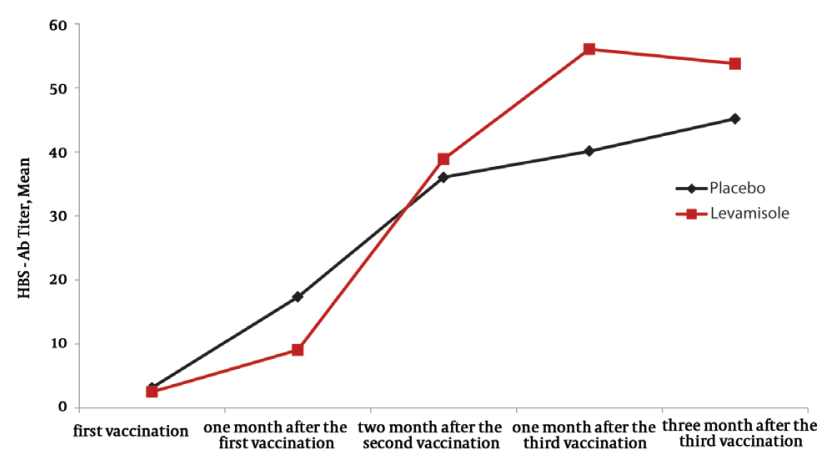

Figure 2. Trend of Antibody Production in the Experimental and Control Groups

life duration in these patients; hence, the importance of chronic infections such as hepatitis $B$ has become more prominent. It seems that preventing HIV infected patients from developing hepatitis B, may result in a serious reduction in the complications that follow this condition and mortality rates, due to the coexistence of the two viruses. As the immune response to HBV vaccination in HIV infected patients is lower than in the normal population, the use of methods which will increase antibody production and result in a more effective immune response following vaccination, is of great importance.

As is shown in Figure 1, the administration of a levamisole adjuvant with HBV vaccination in HIV infected patients after at least two vaccinations, resulted in an increased immune response in this group of patients, in comparison with the control group. This finding is more prominent in subsequent sessions of vaccination, as the $P$-value of the level of immune response decreased toward the level of statistical significance. Such differences in immune response and titer of $\mathrm{Ab}$, became clinically more important one and three months after the third vaccination, however, because some patients were lost to followup, which therefore decreases the power of the study, it did not reach a statistically significant level. There is no doubt though, that the higher immune response in the patients receiving levamisole is clinically very important, but it was not unexpected to find a statistically significant difference $(P<0.05)$ in immune response and antibody production in the experimental group, this may differ if the sample size was larger or the vaccination times were increased. Although using levamisole as an adjuvant for hepatitis B vaccination in HIV infected patients has been paid little attention, there are also limited studies on hepatitis vaccination and the effects of a levamisole adjuvant, inducing an immune response with other diseases. For example, in a study by Arqani et al. in dialysis patients, using a levamisole adjuvant and intradermal injection of hepatitis B vaccine was associated with a higher immune response (14). This was also the case, in a study by Sali et al. concerning the effect of levamisole on the immune response to hepatitis $\mathrm{B}$ vaccination in dialysis patients, it 
was concluded that levamisole had no effect on the level of immune response in this group of patients (19). Kayatas' study on this issue, showed that a levamisole adjuvant increased the immune response to hepatitis B vaccination from $57 \%$ to $82 \%$ (16). In a meta-analysis by Alavian et al. on the effect of oral levamisole as an adjuvant for hepatitis B vaccine in patients with chronic renal failure, it was shown that levamisole increased seroprotection (immune protection) in this group of dialysis patients (17). In our study, patients with CD4 counts greater than $200 \mathrm{cell} / \mathrm{mm} 3$, had a substantially increased production of antibodies, one and three months after completion of vaccination. It seems that a higher CD4 count is associated with a better immune response. Thus, if the study patients in the intervention and control groups were selected from HIV infected patients with CD4 counts of more than 200 cell $/ \mathrm{mm} 3$, it is possible that the intervention with levamisole would have had a significant impact on HBsAb production. Similarly in a study by Cruciani et al. on the immune response to higher doses and more frequent times of HBV vaccination in HIV infected patients, they excluded the patients with CD4 counts less than 200 cell/mm3 and their study showed that the patients with higher CD4 counts have better responses to higher doses and more frequent hepatitis B vaccination (20). Also in the study by Sasaki et al. the effect of granulocyte-monocyte colony-stimulating factor as the adjuvant to hepatitis B vaccine was evaluated in HIV infected patients, the patients with CD4 counts less than 350 cell $/ \mathrm{mm} 3$ were excluded and thus a statistically significant difference between CD4 count and antibody production was not observed in the studied patients (10). Moreover, Fonseca et al. who studied a doubled dose of HBV vaccine in HIV infected patients, recognized that the patients with CD4 counts of more than $350 \mathrm{cell} / \mathrm{mm} 3$ showed a higher seroconversion level with the doubled dose, while there was no difference between vaccination dosage and immune response in the patients with $\mathrm{CD} 4$ counts of less than $350 \mathrm{cell} / \mathrm{mm} 3$ (12). On the other hand, Cooper et al. who studied the effect of vaccination with adjuvant CPG7909 in HIV infected patients excluded those with CD4 counts less than 200 cell $/ \mathrm{mm} 3$ from the study and thus did not observe a significant difference in the CD4 counts between the study groups (1). In a study by Cornejo-Juarez et al. on the effects of different dosages of hepatitis B vaccine on HIV infected patients, it was concluded that because their study included patients with CD4 counts of less than 200 cell $/ \mathrm{mm} 3$, the effect of higher doses of vaccine was not recognizable. They suggested that vaccination should be considered in HIV infected patients with a CD4 count higher than 200 cell/mm3 (9). However, it is concluded from our study that the use of levamisole in HIV infected patients is associated with a better immune response (90\% in the levamisole group vs. $65.38 \%$ in the placebo group, three months after completion of vaccination), this desirable effect was more prominent in the patients with a CD4 count higher than 200 cell/mm3.

In conclusion, our study contained some limitations. The number of HIV infected patients who had not been infected by HBV and had not previously been vaccinated against HBV was small in our study. On the other hand, the number of eligible patients who left the study due to disease complications and personal and social problems was unexpectedly high, resulting in difficulties in gathering enough patients for the desirable study sample size and, hence, achieving significant statistical results. Designing multi-centric studies, as well as increasing the duration of the research, when the number of subjects is limited would increase the power of these types of studies.

\section{Acknowledgments}

The authors would like to thank Dr. Khademi and Mr. Komasi for their kind cooperation.

\section{Authors' Contribution}

Babak Sayad, Seyyed Moayed Alavian and Bita Soltani designed the study and were responsible for overall study management. Farid Najafi did the analysis. Babak Sayad and Bita Soltani prepared the manuscript. All authors contributed to the final version of the manuscript.

\section{Financial Disclosure}

None Declared.

\section{Funding/Support}

This study is the result of a medical student thesis to take a general physician degree from Kermanshah University of Medical Science by Miss. Bita Soltani.

\section{References}

1. Cooper CL, Davis HL, Angel JB, Morris ML, Elfer SM, Seguin I, et al. CPG 7909 adjuvant improves hepatitis B virus vaccine seroprotection in antiretroviral-treated HIV-infected adults. AIDS. 2005;19(14):1473-9.

2. Janbakhsh A, Vaziri S, Sayad B, Afsharian M, Rezaei M, Montazeripour H. Immune Response to Standard Dose of Hepatitis B Vaccine in HIV Positive Clients of Kermanshah Behavioral Diseases Counseling Center. Hepat Mon. 2006;6(2):71-4.

3. Ungulkraiwit P, Jongjirawisan Y, Atamasirikul K, Sungkanuparph S. Factors for predicting successful immune response to hepatitis B vaccination in HIV-1 infected patients. Southeast Asian J Trop Med Public Health. 2007;38(4):680-5.

4. Fauci AS, Lane HC. Human immunodeficiency virus disease: AIDS and related disorders. In: Fauci AS, Braunwald E, Hauser SL, Longo DL, Jameson JL, Joseph L, editors. Harrison's principles of internal medicine. New York: McGraw-Hill; 2008.

5. Overton ET, Kang M, Peters MG, Umbleja T, Alston-Smith BL, Bastow B, et al. Immune response to hepatitis B vaccine in HIVinfected subjects using granulocyte-macrophage colony-stimulating factor (GM-CSF) as a vaccine adjuvant: ACTG study 5220. Vaccine. 2010;28(34):5597-604.

6. Bailey CL, Smith V, Sands M. Hepatitis B vaccine: a seven-year study of adherence to the immunization guidelines and efficacy in HIV-1-positive adults. Int J Infect Dis. 2008;12(6):e77-83. 
7. AMIN S, Andalibi S, MAHMOUDI M. Anti-HBs response and its protective effect in children and adults receiving hepatitis B recombinant vaccine in Tehran. IJMS. 2002;27(3):101-5.

8. Aminzadeh Z, Akhavan H, Gachkar L. Source and response of Antibody to hepatitis B Vaccine in hemodialysis patients. Hepat Mon. 2007;7(1):33-4.

9. Cornejo-Juarez P, Volkow-Fernandez P, Escobedo-Lopez K, VilarCompte D, Ruiz-Palacios G, Soto-Ramirez LE. Randomized controlled trial of Hepatitis B virus vaccine in HIV-1-infected patients comparing two different doses. AIDS Res Ther. 2006;3:9.

10. Sasaki MG, Foccacia R, de Messias-Reason IJ. Efficacy of granulocyte-macrophage colony-stimulating factor (GM-CSF) as a vaccine adjuvant for hepatitis B virus in patients with HIV infection. Vaccine. 2003;21(31):4545-9.

11. Barraclough KA, Wiggins KJ, Hawley CM, van Eps CL, Mudge DW, Johnson DW, et al. Intradermal versus intramuscular hepatitis B vaccination in hemodialysis patients: a prospective open-label randomized controlled trial in nonresponders to primary vaccination. Am J Kidney Dis. 2009;54(1):95-103.

12. Fonseca MO, Pang LW, de Paula Cavalheiro N, Barone AA, Heloisa Lopes M. Randomized trial of recombinant hepatitis B vaccine in HIV-infected adult patients comparing a standard dose to a double dose. Vaccine. 2005;23(22):2902-8.

13. Chow KM, Lo SH, Szeto CC, Yuen SK, Wong KS, Kwan BC, et al. Extra-high-dose hepatitis B vaccination does not confer longer serological protection in peritoneal dialysis patients: a randomized controlled trial. Nephrol Dial Transplant. 2010;25(7):2303-9.
14. Potsch DV, Oliveira ML, Ginuino C, Miguel JC, Oliveira SA, Silva EF, et al. High rates of serological response to a modified hepatitis B vaccination schedule in HIV-infected adults subjects. Vaccine. 2010;28(6):1447-50.

15. Demirci F, Bayraktaroglu Z, Karaoglan M, Coskun Y, Karaoglan I, Okan V. Immunomodulatory effects of HBsAg vaccine and levamisole in chronic hepatitis B and hepatitis B carrier children. Turk J Gastroenterol. 2005;16(4):188-93.

16. Kayatas M. Levamisole treatment enhances protective antibody response to hepatitis B vaccination in hemodialysis patients. Artif Organs. 2002;26(6):492-6.

17. Alavian SM, Tabatabaei SV. Effects of oral levamisole as an adjuvant to hepatitis B vaccine in adults with end-stage renal disease: a meta-analysis of controlled clinical trials. Clin Ther. 2010;32(1):110.

18. Argani H, Akhtarishojaie E. Levamizole enhances immune responsiveness to intra-dermal and intra-muscular hepatitis B vaccination in chronic hemodialysis patients. J Immune Based Ther Vaccines. 2006;4:3.

19. Sali S, Alavian SM, Hajarizadeh B. Effect of levamisole supplementation on hepatitis B virus vaccination response in hemodialysis patients. Nephrology (Carlton). 2008;13(5):376-9.

20. Cruciani M, Mengoli C, Serpelloni G, Lanza A, Gomma M, Nardi $S$, et al. Serologic response to hepatitis B vaccine with high dose and increasing number of injections in HIV infected adult patients. Vaccine. 2009;27(1):17-22. 